\title{
Activation of AMP-activated protein kinase sensitizes lung cancer cells and H1299 xenografts to erlotinib
}

\author{
Helen J. Hülsmann ${ }^{\mathrm{a}, 1}$, Jana Rolff ${ }^{\mathrm{b}, \mathrm{c}, 1}$, Christian Bender $^{\mathrm{d}, 2}$, Mostafa Jarahian ${ }^{\mathrm{e}}$, Ulrike Korf ${ }^{\mathrm{d}}$, \\ Ralf Herwig $^{\mathrm{f}}$, Holger Fröhlich ${ }^{\mathrm{g}}$, Michael Thomas ${ }^{\mathrm{h}, \mathrm{i}}$, Johannes Merk ${ }^{\mathrm{j}, 3}$, Iduna Fichtner ${ }^{\mathrm{b}}$, \\ Holger Sültmann ${ }^{\mathrm{a}, \mathrm{i}, *}$, Ruprecht Kuner ${ }^{\mathrm{a}, \mathrm{i}}$ \\ a Unit Cancer Genome Research, German Cancer Research Center and National Center for Tumor Diseases, Heidelberg, Germany \\ ${ }^{b}$ Experimental Pharmacology, Max-Delbrück-Center, Berlin, Germany \\ ${ }^{\mathrm{c}}$ Experimental Pharmacology \& Oncology Berlin-Buch GmbH, Berlin, Germany \\ d Division of Molecular Genome Analysis, German Cancer Research Center, Heidelberg, Germany \\ e Translational Immunology Unit, German Cancer Research Center and National Center for Tumor Diseases, Heidelberg, Germany \\ ${ }^{\mathrm{f}}$ Vertebrate Genomics Department, Max Planck Institute for Molecular Genetics, Berlin, Germany \\ ${ }^{g}$ University of Bonn, Bonn-Aachen International Center for IT, Bonn, Germany \\ ${ }^{\mathrm{h}}$ Internistische Onkologie der Thoraxtumoren, Thoraxklinik im Universitätsklinikum, Heidelberg, Germany \\ ${ }^{\mathrm{i}}$ Translational Lung Research Center Heidelberg (TLRC-H), Member of the German Center for Lung Research, Heidelberg, Germany \\ j Evangelische Lungenklinik, Berlin, Germany
}

\section{A R T I C L E I N F O}

\section{Article history:}

Received 15 May 2014

Received in revised form 31 July 2014

Accepted 3 September 2014

\section{Keywords:}

EGFR

AMPK

Erlotinib

Lung cancer

Xenograft models

\begin{abstract}
A B S T R A C T
Objectives: The therapeutic scheme for non-small cell lung cancer (NSCLC) patients can be improved if adapted to the individual response. For example, $60-70 \%$ of adenocarcinoma patients show response to EGFR-tyrosine kinase inhibitors in the presence of mutated EGFR. We searched for additional target molecules involved in the action of the EGFR-tyrosine kinase inhibitor erlotinib in the absence of EGFR mutations, which might be suitable for combinatorial therapy approaches.

Materials and Methods: Erlotinib-response associated proteins were investigated in patient-derived NSCLC mouse xenografts by reverse-phase protein array technology (RPPA) and Western blotting. A combinatorial treatment approach was carried out in NSCLC cell lines and H1299 mouse xenografts, and subsequently analyzed for consequences in cell growth and signal transduction.

Results: AMP-activated protein kinase (AMPK) expression was increased in erlotinib responders before and after treatment. In a combinatorial approach, activation of AMPK by A-769662 and erlotinib treatment showed a synergistic effect in cell growth reduction and apoptosis activation in H1299 cells compared to the single drugs. AMPK pathway analyses revealed an effective inhibition of mTOR signaling by drug combination. In H1299 xenografts, the tumor size was significantly decreased after combinatorial treatment.

Conclusion: Our results suggest that AMPK activation status affects response to erlotinib in distinct lung tumor models.
\end{abstract}

(c) 2014 Elsevier Ireland Ltd. All rights reserved.

\footnotetext{
* Corresponding author at: German Cancer Consortium (DKTK), Unit Cancer Genome Research, Division of Molecular Genetics, German Cancer Research Center and National Center for Tumor Diseases, Im Neuenheimer Feld 460, 69120 Heidelberg, Germany. Tel.: +49622156 5934; fax: +496221565382.

E-mail address: h.sueltmann@dkfz.de (H. Sültmann).

1 These authors contributed equally to this work.

2 Present address: TRON, Mainz, Germany.

3 Present address: Sektion Thoraxchirurgie, Klinik für Herz-, Thorax- und Gefäßchirurgie, Universitätsklinikum Ulm, Germany.
}

\section{Introduction}

Recent efforts in lung cancer research to detect driver mutations and novel target sites hold promise to categorize lung cancer patients for suitable therapy regimens. For example, the EGFR-TKI erlotinib represents a potentially effective cancer drug for a subset of lung tumors dependent on the mutation status of EGFR and KRAS [1-3]. However, $30-40 \%$ of patients do not respond to therapy, and most of the Erlotinib responders develop resistance after few months [4]. In the absence of EGFR mutations, $1-8 \%$ of patients respond to erlotinib [5-7]. A better stratification of patients for EGFR-TKI is hampered by the fact that drug sensitivity depends 
on the mutation type of the target and subsequently on the activation status of EGFR downstream pathway and potential bypass signaling [8]. Thus, additional predictive biomarkers are needed to improve patient stratification, and to suggest novel targets for mono- or combination therapy.

AMP-activated protein kinase (AMPK) activators and inhibitors are under investigation as novel cancer drugs [9]. However, the different functions of AMPK signaling suggest an ambivalent role in oncogenic transformation. In general, AMPK is activated by cellular stress like starvation and regulates energy homeostasis [10,11]. Furthermore, AMPK is an upstream regulator of mTOR signaling proposing a tumor suppressor role [12].

In the present study, tumor specimens from twenty-five individual patient-derived lung cancer xenograft models were analyzed. Their drug sensitivity to chemotherapeutics and EGFR inhibitors has been previously characterized [13]. These NSCLC xenografts, all EGFR wild-type, were screened for putative biomarkers and novel drug targets associated with therapy response to EGFR-TKIs. The drug response patterns and protein profiles before and after treatment of NSCLC models suggested AMPK as a putative target protein. Combinatorial treatment including AMPK activators in diverse lung cancer cell lines and a H1299 xenograft model was done to investigate changes in erlotinib sensitivity and tumor growth. Furthermore, the consequences on signal transduction pathways upon single and combinatorial treatment were examined on the protein level.

\section{Materials and methods}

\subsection{NSCLC xenograft models}

Animals were handled according to regulations established by the European Community Council Directive and to protocols approved by the animal care and use committee of the State Office of Health and Social Affairs Berlin (LAGeSo; permit 04520). Details about drug response data, mutation status and NSCLC histology of individual tumors have been previously reported [13]. None of the tumor models harbors an active EGFR mutation.

Xenografts of the NSCLC cell line H1299 were established by subcutaneous implantation of $1 \times 10^{7}$ cells to female NMRI:nu/nu mice (6-8 weeks old). At palpable tumor size $\left(75-100 \mathrm{~mm}^{3}\right)$ five mice each were randomly distributed to three treatment and one control group. Treatment was initiated at day 7 following the scheme: A769662 (Selleck Chemicals Co., Ltd., Houston, USA), 30 mg/kg, QD5, i.p. or erlotinib (Hoffmann-LaRoche, Grenzach-Wyhlen, Germany), $50 \mathrm{mg} / \mathrm{kg}$, QD5, p.o. as monotherapy and in combination therapy. Tumor size was measured in two dimensions twice weekly for 34 days.

\subsection{Reverse Phase Protein Arrays (RPPA)}

Xenograft tissues (cell lines) were lysed in T-PER buffer (MPER buffer) (Thermo Fisher Scientific, Waltham, USA) including $2 \mu \mathrm{M}$ staurosporine, $1 \times$ PhosStop phosphatase and $1 \times$ Mini Complete EDTA-free protease inhibitor cocktails (Roche, Mannheim, Germany). Proteins were quantified by BCA assay (Thermo Fisher Scientific). RPPA spotting was carried out as previously described [14]. After blocking, slides were incubated for $2 \mathrm{~h}$ at RT with primary antibodies (Supplementary Table S1). Antibody-mediated signal amplification (AMSA) was used for signal detection [15]. The average intensity of each spot was determined using GenePix Pro 5.0 software (Molecular Devices, Ismaning, Germany). Data normalization was done using total protein intensity determined by Fast green FCF staining. Data were quality checked relying on protein lysate dilution curve data and protein signal intensities.
Spearman's rank correlation and correlation test statistics was used to compare protein expression with the drug response patterns of the xenografts $(T / C$ values). Proteins were ranked according to the $p$-value without adjustment $(p<0.05)$.

\subsection{Western blotting}

$20 \mu \mathrm{g}$ protein was separated by $6-12 \%$ SDS-PAGE gels and blotted to PVDF membranes using the Trans-Blot SD Semi-Dry Transfer Cell (Bio-Rad, Hercules, CA, USA). After blocking, the blots were incubated with primary antibodies (Supplementary Table S1) overnight at $4{ }^{\circ} \mathrm{C}$. Conjugated secondary goat anti-mouse (antirabbit) IgG DyLightTM680 (800) antibodies were incubated for $2 \mathrm{~h}$. Signals were detected using the Odyssey ${ }^{\circledR}$ Infrared Imaging System (LI-COR Biosciences).

\subsection{Cell culture and drug treatment}

The human NSCLC cell lines H1299, A549 and H1650 were purchased from ATCC (Manassas, VA). The cell lines were authenticated and single mutations were confirmed in A549 (KRAS, G12S), H1299 (NRAS, Q61K), and H1650 (EGFR, E746 A750del) using MassARRAY technology (OncoCarta v1.0 Genotyping, Sequenom, San Diego, CA; Supplementary Table S2). H1299 and H1650 cells were cultured in RPMI-1640 medium, A549 cells in Ham's F-12 K medium, with $10 \%$ FBS (Life Technologies, Carlsbad, CA). The cells were seeded on 96 -well plates (6-well plates) at densities between 1 and $2 \times 10^{3}$ $\left(0.5\right.$ and $\left.2 \times 10^{5}\right)$ cells per well. Cells were treated with erlotinib (Tarceva, Roche), A-769662 (Selleck Chemicals Co., Ltd., Houston, USA), metformin (Enzo Life Sciences, Farmingdale, USA) and AICAR (Cayman Chemical Company, USA) at different concentrations.

\subsection{Cell viability (WST-1 assay, cell counting)}

Cell viability was analyzed using the "Cell Proliferation Reagent" (WST-1, Roche). Absorbance was measured using a microplate reader (Infinite M200, TECAN, Männedorf, Switzerland). All treatments were carried out in two independent experiments, each with six technical replicates. Cells were counted using an improved Neubauer hemocytometer.

\subsection{FACS analysis (FACS-PI)}

Cells were harvested by Accutase (Life Technologies) treatment, washed with PBS, fixed in $71 \%$ ethanol for at least $24 \mathrm{~h}$ at $-20^{\circ} \mathrm{C}$, treated with RNase A and stained with propidium iodide. DNA fragmentation was determined using FACS-PI (Propidium iodide). The subG1 DNA content was determined to define apoptotic cells. Cytofluorometric analyses were performed using the BD FACSCanto ${ }^{\mathrm{TM}}$ II flow cytometer with BD FACSDiva ${ }^{\mathrm{TM}}$ software v. 6.1.3 (Becton Dickinson, Heidelberg, Germany). FACS analyses were carried out in two independent experiments each with two technical replicates 72 and $120 \mathrm{~h}$ after treatment.

\section{Results}

\subsection{Identification of proteins associated with erlotinib therapy response}

Reverse-phase protein analyses were conducted from tumor protein lysates of 25 untreated xenografts. Raw data from four samples were excluded from further analysis for quality reasons. Normalized RPPA data are summarized in Supplementary Table S3. Spearman's correlation between normalized protein expression values and erlotinib (or cetuximab) response indicated a 
Table 1

Protein expression associated with Erlotinib response in xenograft models.

\begin{tabular}{|c|c|c|c|c|c|c|}
\hline \multirow[t]{2}{*}{ Protein } & \multirow[t]{2}{*}{ Detected Phospho-Site } & \multirow[t]{2}{*}{ Symbol } & \multicolumn{2}{|c|}{ Erlotinib response } & \multicolumn{2}{|c|}{ Cetuximab response } \\
\hline & & & $\mathrm{rs}^{\mathrm{a}}$ & $p$-value & rs & $p$-value \\
\hline RAF1 & None & RAF1 & -0.6980 & 0.0004 & $n s$ & $n s$ \\
\hline P-AMPK alpha & Thr172 & PRKAA1, PRKAA2 & -0.6290 & 0.0022 & -0.5500 & 0.0099 \\
\hline P-C-RAF & S289, S296, S301 & RAF1 & -0.5970 & 0.0043 & $n s$ & $n s$ \\
\hline S6K-alpha-3 & None & RPS6KA3 & -0.5590 & 0.0084 & $n s$ & ns \\
\hline P-c-RAF & S259 & RAF1 & -0.5530 & 0.0093 & $n s$ & ns \\
\hline PDK1 & None & PDK1 & -0.5330 & 0.0129 & $n s$ & $n s$ \\
\hline TGFB & None & TGFB1 & -0.5200 & 0.0158 & $n s$ & $n s$ \\
\hline $\mathrm{p} 21$ & None & CDKN1A & 0.5160 & 0.0167 & $n s$ & $n s$ \\
\hline STAT5 & None & STAT5 & -0.5080 & 0.0187 & $n s$ & $n s$ \\
\hline STAT1 & None & STAT1 & -0.4840 & 0.0262 & ns & $n s$ \\
\hline RRM1 & None & RRM1 & -0.4750 & 0.0294 & $n s$ & ns \\
\hline STK4 & None & STK4 & -0.4750 & 0.0294 & $n s$ & ns \\
\hline STAT3 & None & STAT3 & -0.4570 & 0.0371 & $n s$ & ns \\
\hline P70S6K1 & None & RPS6KB1 & -0.4510 & 0.0400 & $n s$ & $n s$ \\
\hline P-mTOR & Ser2448 & MTOR & $n s$ & $n s$ & -0.4840 & 0.0262 \\
\hline ERbB4 & None & ERBB4 & $n s$ & $n s$ & -0.4510 & 0.0400 \\
\hline
\end{tabular}

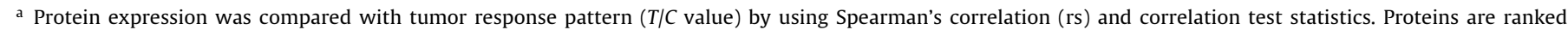
according to the unadjusted $p$-value ( $n s \geq 0.05$ ).

potential association for 16 out of 77 analyzed proteins in this dataset (Table 1). Higher expression of phosphorylated $\left(\mathrm{Thr}^{172}\right)$ AMP-activated protein kinase (AMPK) alpha correlated with a better response to erlotinib ( $\mathrm{rs}=-0.63$ ) and cetuximab $(\mathrm{rs}=-0.55)$ (Supplementary Fig. S1). The expression of AMPK and P-AMPK was further investigated by Western blotting in eight xenograft tumors with and without erlotinib-treatment (Fig. 1). In the three best responders (T/C value 26-31), the expression of AMPK and P-AMPK was increased after erlotinib treatment, which was not obvious in erlotinib non-responders ( $T / C$ value 76-97). Weak expression of the proteins was shown for two non-responders (7747, 7433). One non-responder (7187) had equal target protein expression with and without erlotinib exposure. In one model (7166) AMPK expression strongly decreased upon erlotinib treatment indicating a change in the signaling cascade of this non-responder.

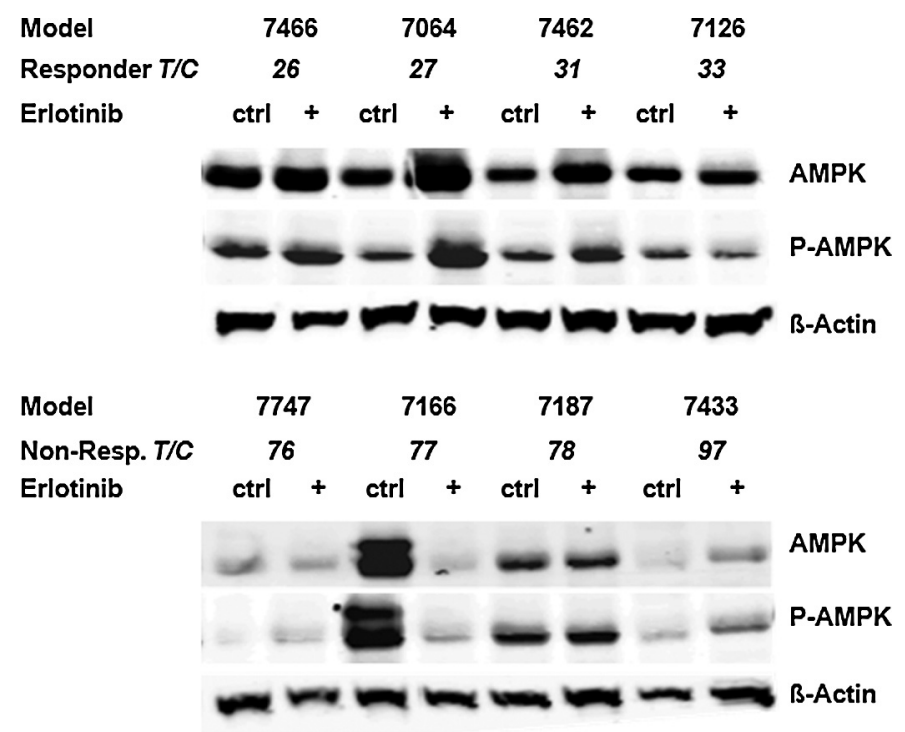

Fig. 1. Western blot analysis of Erlotinib-treated and control xenograft models. Xenograft models are characterized by erlotinib response pattern (Responder top, Non-Responder bottom) based on $T / C$ values. All eight xenograft models were treated with erlotinib and normal saline (control). The abundance of the proteins AMPK and P-AMPK was determined; $\beta$-Actin staining was used as loading control.

\subsection{A-769662 treatment activates AMPK in H1299 cells in a dose-dependent manner}

Three NSCLC cell lines were treated with different concentrations of A-769662, a known activator of AMPK signaling. First, protein expression changes were screened by RPPA analysis. In H1299 cells, RPPA data indicated P-AMPK and PRKCA increase upon A-769662 treatment. In A549 cells, Caveolin-1 and P-AcetylCoA (ACC) showed a higher abundance. In H1650 cells, E2F2 was increased, and P-RB and P-S6 were decreased upon A-769662 treatment (Supplementary Table S4). For P-AMPK, lowest endogenous protein levels were observed in H1299 cells based on RPPA and Western blotting (Fig. 2). Increased P-AMPK protein levels dependent on elevated A-769662 concentrations were detectable in H1299 cells. H1650 cells showed a non-linear P-AMPK increase after A-769662 treatment, A549 cells did not show any changes.

\subsection{Combinatorial erlotinib and A-769662 treatment decreases cell viability and activates apoptosis in $\mathrm{H} 1299$ cells}

The sensitivity of three NSCLC cell lines was determined for A-769662 and erlotinib. For A-769662, drug sensitivity (IC50: $150-400 \mu \mathrm{M})$ decreased from H1299, H1650 to A549 cell line (Fig. 3A). For erlotinib, drug sensitivity was highest in H1650 cells compared to A549 and H1299, as previously reported [16]. Combinatorial treatment was most effective in H1299 (50-65\% cell viability reduction), marginally effective in A549 (10-25\%), and not beneficial in $\mathrm{H} 1650$ cells (Fig. 3B). Cell counting in the same experimental setting confirmed the viability decrease in H1299 and A549 cells after combinatorial treatment (Supplementary Fig. S2).

H1299 cells were treated with $5,7.5$ or $10 \mu \mathrm{M}$ erlotinib alone or in combination with 15,45 or $75 \mu \mathrm{M}$ A-769662. Cell viability was constantly decreased in H1299 cells after combined A-769662 and erlotinib treatment $(72 \mathrm{~h}$ ) in comparison with single drugs and DMSO controls (Fig. 3C). A-769662 alone had a marginal effect on cell viability (0-10\%), erlotinib alone slightly decreased cell viability (7-28\%) compared with DMSO control (100\%). In contrast, combinatorial treatment using A-769662 and erlotinib strongly inhibited cell viability (52-76\%), with the strongest inhibitory effect at the highest concentrations ( $75 \mu \mathrm{M}$ A-769662 and $10 \mu \mathrm{M}$ erlotinib). H1299 cell growth between 0 and $72 \mathrm{~h}$ supported the additive combinatorial effects of the A-769662 and erlotinib combination (Supplementary Fig. S3). 
$\mathrm{H} 1299$

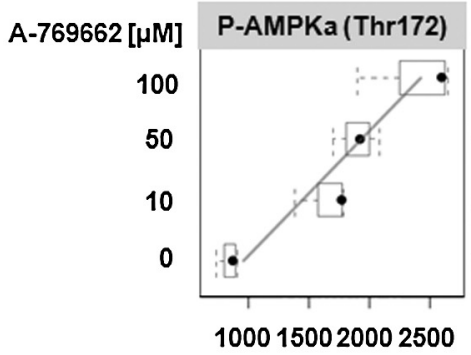

A549

1600180020002200

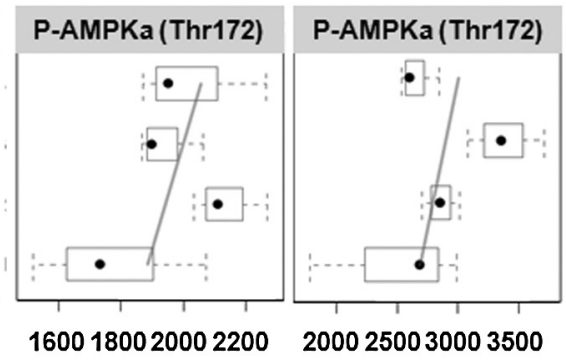

$\mathrm{H} 1650$

Signal

intensity

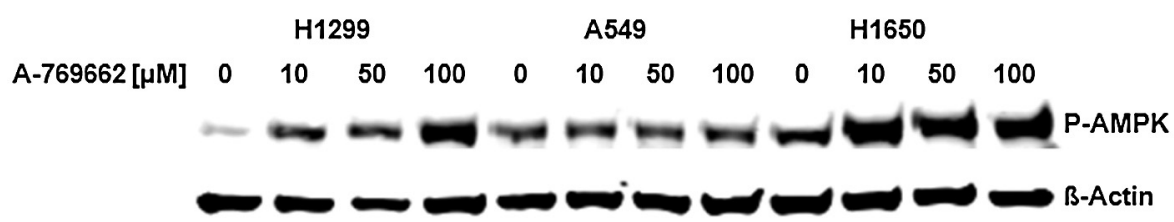

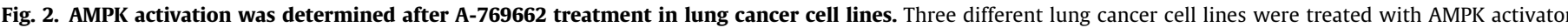
A-769662 (0, 10, 50 and $100 \mu \mathrm{M})$. Proteins were measured by RPPA (P-AMPK) and Western blotting (P-AMPK and $\beta$-Actin loading control).

SubG1 FACS detection (PI-staining) of $\mathrm{H} 1299$ cells upon $72 \mathrm{~h}$ and 120 h combinatorial treatment ( $10 \mu \mathrm{M}$ erlotinib, $75 \mu \mathrm{M}$ A-769662) was carried out. We detected the strongest increase of apoptotic (subG1) cells in cultures treated with both drugs (21\%) in comparison to single drugs (4-7\%) and DMSO solvent control (3\%) (Fig. 3D). This indicated that combinatorial treatment with erlotinib and A769662 activates apoptosis in H1299 cells.

To further corroborate the combinatorial effect, treatment was performed in $\mathrm{H} 1299$ cells with $10 \mu \mathrm{M}$ erlotinib and the AMPK activators metformin $(1 \mathrm{mM})$ or AICAR $(50 \mu \mathrm{M})$. AMPK activation by these compounds also had a positive effect on erlotinib sensitivity in H1299 cells: we observed residual cell viability of 56\% for metformin and of $71 \%$ for AICAR (Supplementary Fig. S4).

\subsection{Combinatorial A-769662 and erlotinib treatment of H1299 cells inhibits $m$ TOR signaling}

To identify the molecular consequences of AMPK activation and erlotinib treatment on cellular signaling, Western blot analysis of H1299 protein lysates after single and combinatorial treatment was carried out (Fig. 4). P-AMPK levels differed in particular treatment settings between both time points. After $24 \mathrm{~h}$, AMPK, P-AMPK and downstream P-ACC levels increased after combinatorial treatment indicating activated AMPK signaling. P-mTOR did not differ between single and combinatorial treatment. In contrast, P-Raptor, whose phosphorylation inhibits the mTOR complex 1 (mTORC1), increased in the presence of AMPK activator A-769662.
A

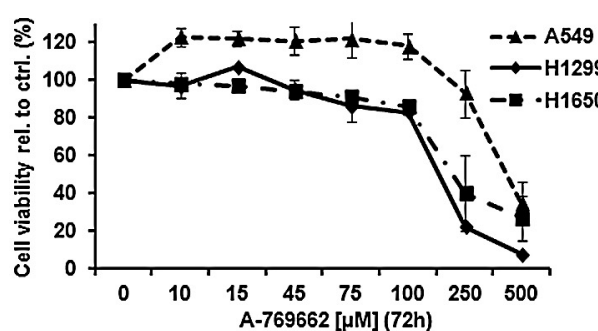

C

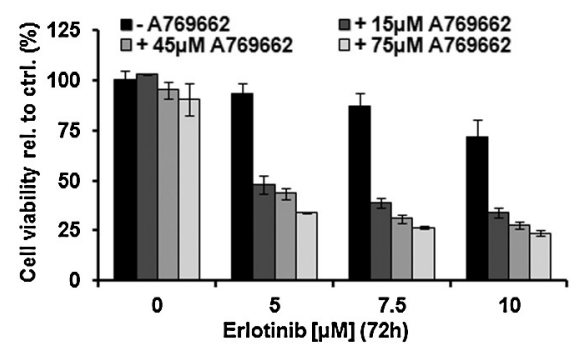

B

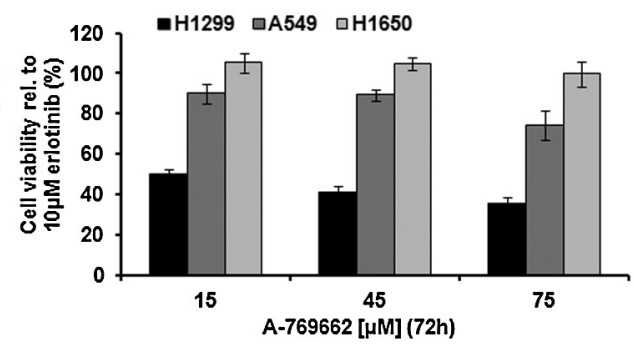

D

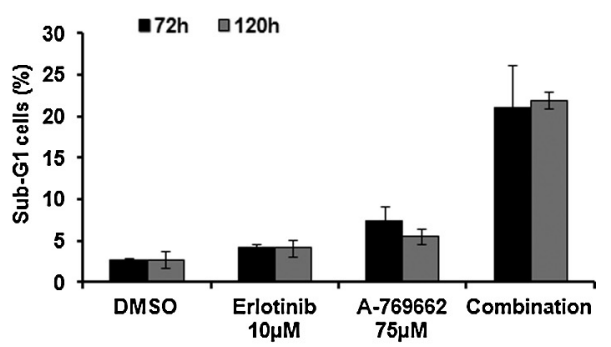

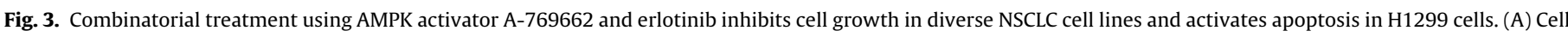

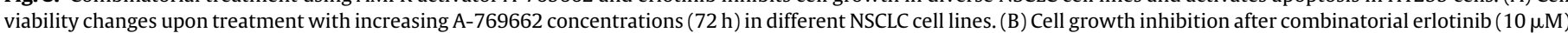

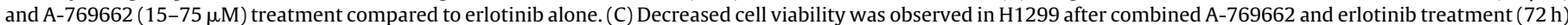

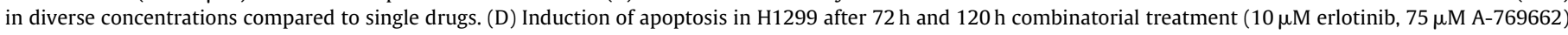
was detected by subG1 FACS detection (PI-staining). 


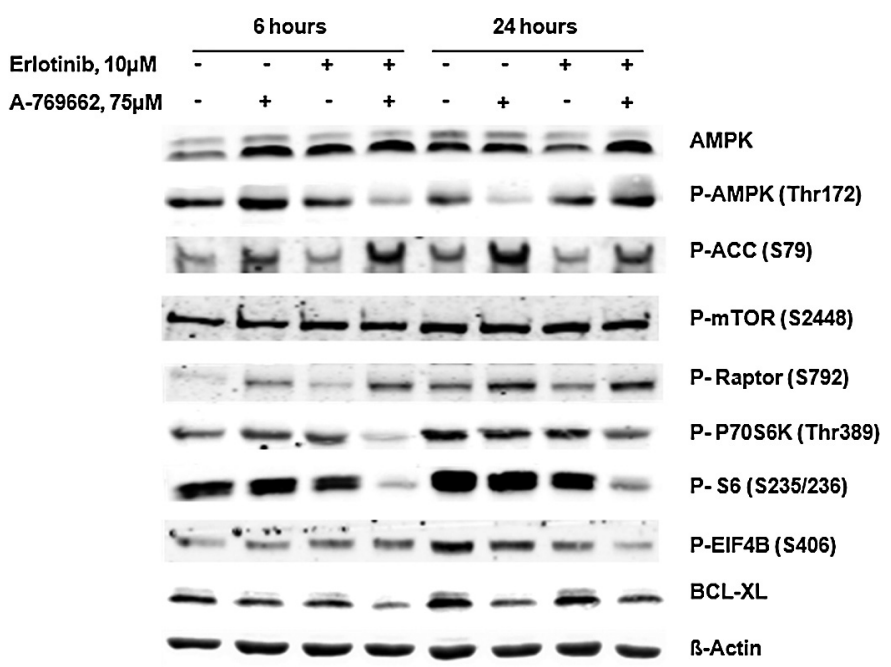

Fig. 4. Combinatorial treatment of $\mathrm{H1299}$ cells with A-769662 and erlotinib strongly inhibited signaling proteins like p70s6K and S6. Proteins associated with AMPK and mTOR signaling were analyzed 6 and $24 \mathrm{~h}$ after treatment (DMSO, A769662 , erlotinib, and combination) of H1299 cells. $\beta$-Actin was used as loading control.

The strongest differences were detected for the mTOR downstream proteins P-P70S6K and P-S6: their levels strongly decreased in the cells treated with both compounds only ( 6 and $24 \mathrm{~h}$ ). Inhibition of P-EIF4B, a downstream protein of P70S6K, was most evident after combinatorial treatment $(24 \mathrm{~h})$. The levels of anti-apoptotic protein BCL-XL in the presence of A-769662 (24 h), which might explain the observed induction of apoptosis in $\mathrm{H} 1299$ cells in the combinatorial treatment. Autophagic cell death was investigated by the conversion of LC3-I into LC3-II protein fraction. In our model, A-769662 alone did not induce LC3 conversion (Supplemental Fig. S5). LC3-II fraction and autophagy was induced upon erlotinib treatment (48 and $72 \mathrm{~h}$ ). This effect was reversible upon combinatorial treatment. In summary, the combinatorial treatment had a strong inhibitory impact on mTOR downstream signaling compared to AMPK activation or erlotinib treatment alone.

\subsection{Combinatorial treatment decreases tumor growth in a H1299 mouse xenograft model}

The growth inhibitory effects of AMPK activation and erlotinib combinatorial treatment were further explored in vivo using human H1299 NSCLC xenografts in mice. The mice were treated for 5 days (day 7-11) either with a single drug (erlotinib or A-769662), in combination or saline control. Subcutaneous tumor growth (five mice per condition) was measured in vivo for 34 days. Finally, the tumors were surgically removed. The combination of erlotinib and A-769662 decreased tumor size significantly in comparison to control and single treatment ( $p=0.03$; Fig. $5 A$ ). Resected tumors (three replicates closest to median size) from different treatment regimens are shown to demonstrate growth response. The combination therapy had the strongest impact on tumor growth in

A
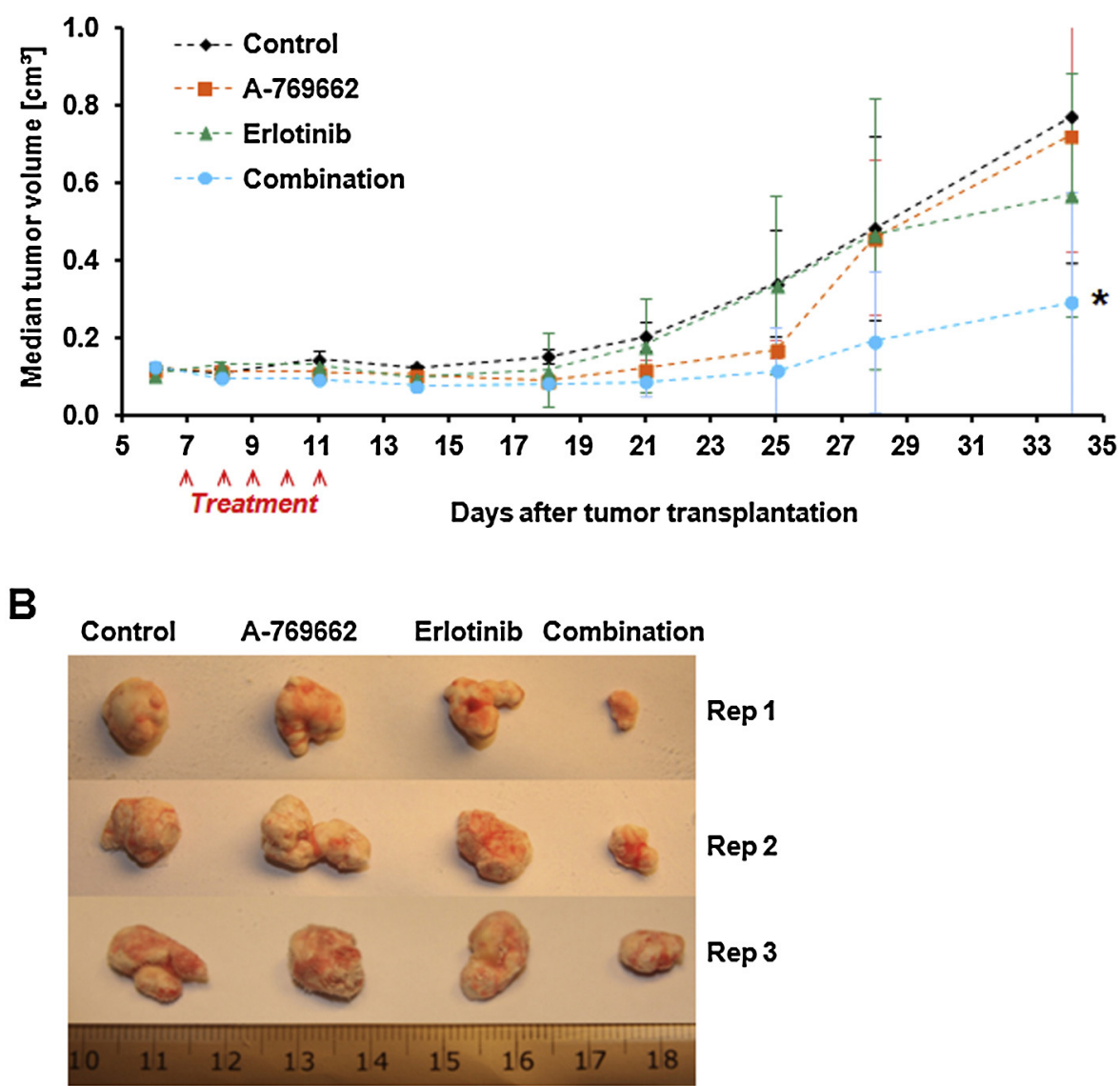

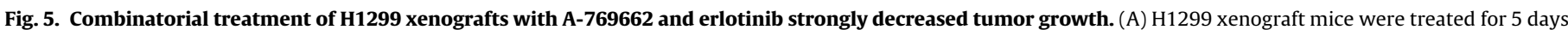

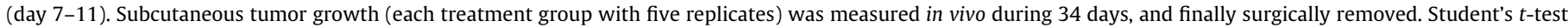

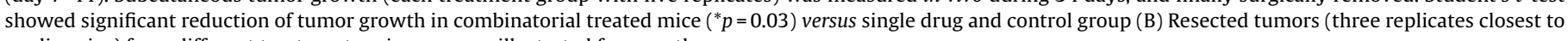
median size) from different treatment regimens were illustrated for growth response. 
H1299 xenografts. No significant differences in tumor size were observed for the other experimental conditions (Fig. 5B). These findings suggested that AMPK activation enhances the antitumor action of erlotinib in vivo.

\section{Discussion}

EGFR mutations and the EML4-ALK gene fusion represent two molecular alterations in about $15 \%$ of lung adenocarcinoma patients in Western countries, and affect target sites for which drugs are in clinical use [17]. The benefit for patients treated with erlotinib and gefitinib was only significant when stratified according to EGFR mutation status $[18,19]$. However, about $30-40 \%$ of patients do not respond to EGFR inhibitors using the current selection criteria $[1,20]$. About $1-8 \%$ of patients without relevant EGFR mutations are responders (Mok, NEJM, 2009; Janne, JCO, 2012; Garassino, Lancet, 2013). The complexity of genomic alterations in lung tumors may determine these limitations. For example, the role of a KRAS mutation as negative predictor for EGFR-targeted therapy is controversially discussed [21]. It is likely that the activity of additional proteins affects the sensitivity against EGFR-targeted therapies in an individual patient.

In this study, NSCLC xenograft models were screened to identify molecules associated with therapy response to the EGFR-targeted therapeutics, erlotinib and cetuximab. As a novel finding, P-AMPK expression was elevated in several NSCLC xenograft models which subsequently respond better to erlotinib or cetuximab. In addition, AMPK and P-AMPK expression was increased after erlotinib treatment in the three best responders. These findings suggested that AMPK activation status impacts on EGFR targeted drug response in distinct lung cancer models. In lung cancer, AMPK signaling is connected with important oncogenic pathways, and associated with tumorigenesis and progression. It was shown that metformin, a biguanide anti-diabetic drug and activator of the AMPK signaling, decreases the risk for cancer in diabetes patients [22]. Furthermore, LKB1, a known upstream activator of AMPK signaling, has been described as tumor suppressor in lung cancer cell lines and mouse tumors $[23,24]$.

In our study, AMPK activation using A-769662 sensitizes lung cancer cells to erlotinib treatment. The reduction of cell growth was most convincing in EGFR wild-type H1299 cells, which may due be to the low endogenous AMPK levels, its strongest induction via A-769662, and insensitivity to erlotinib. AMPK activation using alternative compounds like metformin or AICAR confirmed the increased erlotinib sensitivity. Several studies in NSCLC and other cancers have shown that response to EGFR-targeted therapeutics depends on the activation status of mTOR signaling, and can be increased by mTOR-inhibiting agents [25]. An example is the synergistic effect of combinatorial treatment with erlotinib and rapamycin [26]. Moreover, AMPK has an impact on mTOR signaling: AMPK can directly phosphorylate Raptor leading to the inhibition of the mTOR complex 1 and cell cycle arrest [12].

Solely administration of A-769662 in $\mathrm{H} 1299$ raised the protein levels of downstream effectors P-ACC and P-Raptor, but did not affect mTOR downstream targets and cell growth. In contrast, combination of erlotinib and A-769662 strongly decreased cell viability and activated apoptosis in H1299. Apoptosis induction may be due to inhibition of the anti-apoptotic protein BCL-xL upon combinatorial treatment. It has been described that AMPK is required for $B C L-x L$ mRNA destabilization and initiation of intrinsic apoptosis [27]. The observed autophagy-linked LC3 conversion upon erlotinib has been previously reported [28]. However, autophagy is likely not the dominant effect on cell viability upon combinatorial treatment in our study. In contrast to single treatments, combination resulted in a strong reduction of mTOR downstream targets P-P70S6K,
P-S6 and P-EIF4B, which likely induced cell growth inhibition. These findings might be caused by a concerted action of both agents targeting mTOR signaling. In diverse tumor entities EGFR mutational status was associated with phosphorylation of mTOR and S6 $[29,30]$. In our NSCLC model, inhibition of mTOR downstream genes independent of P-mTOR suggests an alternative signal transduction mode via phosphorylation of Raptor.

From three tested NSCLC cell lines, the strongest effects of treatment combination were shown in $\mathrm{H} 1299$ cells. The impact on tumor growth was confirmed in H1299 xenograft models. Possibly, specific mutations influence the sensitivity to this treatment concept. NRAS mutations were found in about $1 \%$ of lung tumors, and cell lines like H1299 harboring NRAS mutation behaved insensitive to EGFR-TKI [31]. In NRAS-mutant melanoma cells treatment with AMPK activator AICAR inhibited cell growth [32]. In A549 cells, PAMPK was hardly activated by A-769662 that is probably due to a loss of functional LKB1 [33]. The detailed crosstalk between EGFR and AMPK signaling concerning dual action on mTOR downstream pathway in the context of specific tumor genome alterations has to be further elucidated.

In conclusion, we observed that AMPK activation using A769662 sensitizes distinct lung cancer cell lines and H1299 xenograft models to erlotinib. The activation status of AMPK signaling in a subset of lung tumors may affect the sensitivity to EGFR-targeted therapies.

\section{Role of the funding source}

This study was supported by the German Federal Ministry of Education and Research BMBF including the grants PREDICT (0315428C), and the BMBF grant German Center for Lung Research (82DZL00404).

\section{Conflict of interest statement}

The authors declare no conflict of interest.

\section{Acknowledgments}

We thank Jennifer Metzger, Sabrina Gerhardt and Nadine Mohr for their expert technical assistance.

\section{Appendix A. Supplementary data}

Supplementary material related to this article can be found, in the online version, at http://dx.doi.org/10.1016/j.lungcan. 2014.09.001.

\section{References}

[1] Eberhard DA, Johnson BE, Amler LC, Goddard AD, Heldens SL, Herbst RS, et al. Mutations in the epidermal growth factor receptor and in KRAS are predictive and prognostic indicators in patients with non-small-cell lung cancer treated with chemotherapy alone and in combination with erlotinib. J Clin Oncol 2005;23:5900-9.

[2] Miller VA, Riely GJ, Zakowski MF, Li AR, Patel JD, Heelan RT, et al. Molecular characteristics of bronchioloalveolar carcinoma and adenocarcinoma, bronchioloalveolar carcinoma subtype, predict response to erlotinib. J Clin Oncol 2008;26:1472-8

[3] Zhou C, Wu YL, Chen G, Feng J, Liu XQ, Wang C, et al. Erlotinib versus chemotherapy as first-line treatment for patients with advanced EGFR mutation-positive non-small-cell lung cancer (OPTIMAL, CTONG-0802): a multicentre, openlabel, randomised, phase 3 study. Lancet Oncol 2011;12:735-42.

[4] Ettinger DS, Akerley W, Borghaei H, Chang AC, Cheney RT, Chirieac LR, et al. Non-small cell lung cancer. J Natl Comprehensive Cancer Netw: JNCCN 2012;10:1236-71

[5] Garassino MC, Martelli O, Broggini M, Farina G, Veronese S, Rulli E, et al. Erlotinib versus docetaxel as second-line treatment of patients with advanced nonsmall-cell lung cancer and wild-type EGFR tumours (TAILOR): a randomised controlled trial. Lancet Oncol 2013;14:981-8. 
[6] Janne PA, Wang X, Socinski MA, Crawford J, Stinchcombe TE, Gu L, et al. Randomized phase II trial of erlotinib alone or with carboplatin and paclitaxel in patients who were never or light former smokers with advanced lung adenocarcinoma: CALGB 30406 trial. J Clin Oncol 2012;30:2063-9.

[7] Mok TS, Wu YL, Thongprasert S, Yang CH, Chu DT, Saijo N, et al. Gefitinib or carboplatin-paclitaxel in pulmonary adenocarcinoma. New Engl J Med 2009;361:947-57.

[8] Martini M, Vecchione L, Siena S, Tejpar S, Bardelli A. Targeted therapies: how personal should we go? Nat Rev Clin Oncol 2012;9:87-97.

[9] Liang J, Mills GB. AMPK: a contextual oncogene or tumor suppressor? Cancer Res 2013;73:2929-35.

[10] Faubert B, Boily G, Izreig S, Griss T, Samborska B, Dong Z, et al. AMPK is a negative regulator of the Warburg effect and suppresses tumor growth in vivo. Cell Metabol 2013:17:113-24.

[11] Kato K, Ogura T, Kishimoto A, Minegishi Y, Nakajima N, Miyazaki M, et al. Critical roles of AMP-activated protein kinase in constitutive tolerance of cancer cells to nutrient deprivation and tumor formation. Oncogene 2002;21:6082-90.

[12] Gwinn DM, Shackelford DB, Egan DF, Mihaylova MM, Mery A, Vasquez DS, et al. AMPK phosphorylation of raptor mediates a metabolic checkpoint. Mol Cell 2008;30:214-26.

[13] Fichtner I, Rolff J, Soong R, Hoffmann J, Hammer S, Sommer A, et al. Establishment of patient-derived non-small cell lung cancer xenografts as models for the identification of predictive biomarkers. Clin Cancer Res 2008;14:6456-68.

[14] Loebke C, Sueltmann H, Schmidt C, Henjes F, Wiemann S, Poustka A, et al. Infrared-based protein detection arrays for quantitative proteomics. Proteomics 2007; 7:558-64.

[15] Brase JC, Mannsperger H, Frohlich H, Gade S, Schmidt C, Wiemann S, et al. Increasing the sensitivity of reverse phase protein arrays by antibody-mediated signal amplification. Proteome Sci 2010;8:36.

[16] Yauch RL, Januario T, Eberhard DA, Cavet G, Zhu W, Fu L, et al. Epithelial versus mesenchymal phenotype determines in vitro sensitivity and predicts clinical activity of erlotinib in lung cancer patients. Clin Cancer Res 2005;11: 8686-98.

[17] Pao W, Chmielecki J. Rational, biologically based treatment of EGFR-mutant non-small-cell lung cancer. Nat Rev Cancer 2010;10:760-74

[18] Paez JG, Janne PA, Lee JC, Tracy S, Greulich H, Gabriel S, et al. EGFR mutations in lung cancer: correlation with clinical response to gefitinib therapy. Science 2004;304:1497-500.

[19] Tsao MS, Sakurada A, Cutz JC, Zhu CQ Kamel-Reid S, Squire J, et al. Erlotinib in lung cancer - molecular and clinical predictors of outcome. N Engl J Med 2005;353:133-44.
[20] Sequist LV, Martins RG, Spigel D, Grunberg SM, Spira A, Janne PA, et al. Firstline gefitinib in patients with advanced non-small-cell lung cancer harboring somatic EGFR mutations. J Clin Oncol 2008;26:2442-9.

[21] Roberts PJ, Stinchcombe TE, Der CI, Socinski MA. Personalized medicine in non-small-cell lung cancer: is KRAS a useful marker in selecting patients for epidermal growth factor receptor-targeted therapy? J Clin Oncol 2010;28:4769-77.

[22] Evans JM, Donnelly LA, Emslie-Smith AM, Alessi DR, Morris AD. Metformin and reduced risk of cancer in diabetic patients. BMJ 2005;330:1304-5.

[23] Ji H, Ramsey MR, Hayes DN, Fan C, McNamara K, Kozlowski P, et al. LKB1 modulates lung cancer differentiation and metastasis. Nature 2007:448:807-10.

[24] Gill RK, Yang SH, Meerzaman D, Mechanic LE, Bowman ED, Jeon HS, et al. Frequent homozygous deletion of the LKB1/STK11 gene in non-small cell lung cancer. Oncogene 2011;30:3784-91.

[25] Bliesath J, Huser N, Omori M, Bunag D, Proffitt C, Streiner N, et al. Combined inhibition of EGFR and CK2 augments the attenuation of PI3K-Akt-mTOR signaling and the killing of cancer cells. Cancer Lett 2012;322:113-8.

[26] Buck E, Eyzaguirre A, Brown E, Petti F, McCormack S, Haley JD, et al. Rapamycin synergizes with the epidermal growth factor receptor inhibitor erlotinib in nonsmall-cell lung, pancreatic, colon, and breast tumors. Mol Cancer Therapeut 2006;5:2676-84.

[27] Day RM, Lee YH, Han L, Kim YC, Feng YH. Angiotensin II activates AMPK for execution of apoptosis through energy-dependent and -independent mechanisms. Am J Physiol - Lung Cell Mol Physiol 2011;301:L772-81.

[28] Li YY, Lam SK, Mak JC, Zheng CY, Ho JC. Erlotinib-induced autophagy in epidermal growth factor receptor mutated non-small cell lung cancer. Lung Cancer 2013;81:354-61.

[29] Dobashi Y, Suzuki S, Kimura M, Matsubara H, Tsubochi H, Imoto I, et al. Paradigm of kinase-driven pathway downstream of epidermal growth factor receptor/Akt in human lung carcinomas. Hum Pathol 2011;42:214-26.

[30] Fan QW, Cheng C, Knight ZA, Haas-Kogan D, Stokoe D, James CD, et al. EGFR signals to mTOR through PKC and independently of Akt in glioma. Sci Signal 2009;2:ra4

[31] Ohashi K, Sequis LV, Arcila ME, Lovly CM, Chen X, Rudin CM, et al. Characteristics of Lung Cancers Harboring NRAS Mutations. Clin Cancer Res 2013.

[32] Petti C, Vegetti C, Molla A, Bersani I, Cleris L, Mustard KJ, et al. AMPK activators inhibit the proliferation of human melanomas bearing the activated MAPK pathway. Melanoma Res 2012;22:341-50.

[33] Sanchez-Cespedes M, Parrella P, Esteller M, Nomoto S, Trink B, Engles JM, et al. Inactivation of LKB1/STK11 is a common event in adenocarcinomas of the lung. Cancer Res 2002;62:3659-62 